\title{
Gastroesophageal reflux disease, functional dyspepsia and irritable bowel syndrome: common overlapping gastrointestinal disorders
}

\author{
Nicola de Bortolia , Salvatore Tolone ${ }^{\mathrm{b}}$, Marzio Frazzonic, Irene Martinuccid, Giulia Sgherria , Eleonora Albano ${ }^{a}$, \\ Linda Ceccarellia, Cristina Stasi ${ }^{e}$, Massimo Bellinia , Vincenzo Savarino', Edoardo V. Savarino", Santino Marchia \\ University of Pisa; University of Campania Luigi Vanvitelli, Caserta; Baggiovara Hospital, Modena; Versilia Hospital, Lido di Camaiore-Lucca; \\ Careggi University Hospital, Florence; University of Genoa; University of Padua, Italy
}

\begin{abstract}
Several studies have indicated an overlap between gastroesophageal reflux disease (GERD) and various functional gastrointestinal disorders (FGIDs). The overlapping conditions reported have mainly been functional dyspepsia (FD) and irritable bowel syndrome (IBS). The available literature is frequently based on symptomatic questionnaires or endoscopic procedures to diagnose GERD. Rarely, among patients with heartburn, pathophysiological evaluations have been considered to differentiate those with proven GERD from those without. Moreover, both GERD and IBS or FD showed enormous heterogeneity in terms of the criteria and diagnostic procedures used. The GERD-IBS overlap ranges from 3-79\% in questionnaire-based studies and from 10-74\% when GERD has been diagnosed endoscopically. The prevalence of functional dyspepsia (after normal upper endoscopy) is $12-15 \%$ and an overlap with GERD has been reported frequently. Only a few studies have considered a potential overlap between functional heartburn (FH) and IBS using a 24-h pH-metry or impedance-pH evaluation. Similar data has been reported for an overlap between FH and FD. Recently, a revision of the Rome criteria for esophageal FGIDs identified both FH and hypersensitive esophagus (HE) as potential functional esophageal disorders. This might increase the potential overlap between different FGIDs, with FH and HE rather than with GERD. The aim of the present review article was to appraise and discuss the current evidence supporting the possible concomitance of GERD with IBS and FD in the same patients and to evaluate how various GERD treatments could impact on the quality of life of these patients.
\end{abstract}

Keywords Gastroesophageal reflux disease, irritable bowel syndrome, functional dyspepsia, symptomatic questionnaires, proton pump inhibitor

Ann Gastroenterol 2018; 31 (6): 1-10

\begin{abstract}
${ }^{\mathrm{a}}$ Gastroenterology Unit, Department of Translational Research and New Technologies in Medicine and Surgery, University of Pisa (Nicola de Bortoli, Giulia Sgherri, Eleonora Albano, Linda Ceccarelli, Massimo Bellini, Santino Marchi); 'burgery Unit, Department of Surgery, University of Campania Luigi Vanvitelli, Caserta (Salvatore Tolone); 'Division of Pathophysiology, Baggiovara Hospital, Modena (Marzio Frazzoni); ${ }^{\mathrm{D} D i v i s i o n}$ of Gastroenterology, Versilia Hospital, Lido di

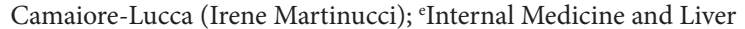
Unit, Department of Experimental and Clinical Medicine, Careggi University Hospital, Florence (Cristina Stasi); ${ }^{\mathrm{f}}$ Gastroenterology Unit, Department of Internal Medicine, University of Genoa (Vincenzo Savarino); ${ }^{\mathrm{g}}$ Gastroenterology Unit, Department Surgery, Oncology and Gastroenterology, University of Padua (Edoardo V. Savarino), Italy
\end{abstract}

\section{Conflict of Interest: None}

Correspondence to: Nicola de Bortoli, MD, Gastroenterology Unit, Department of Translational Research and New Technologies in Medicine and Surgery, University of Pisa, 54124 Pisa, Italy, e-mail: nicola.debortoli@unipi.it

Received: 11 January 2018; accepted 26 August 2018; published online 26 September 2018

DOI: https://doi.org/10.20524/aog.2018.0314

\section{Introduction}

Functional gastrointestinal disorders (FGIDs), according to Rome IV criteria, are defined as variable combinations of chronic or recurrent gastrointestinal signs and symptoms without structural or biochemical alterations [1]. The overall prevalence burden is estimated to be approximately one third of the population [2]. These disorders have been largely studied during the last decades and are thought to occur after gut-brain interaction. Symptoms are generated based on a complex interaction among factors such as microbial dysbiosis within the gut, altered mucosal immune function, altered gut signaling (visceral hypersensitivity) and central nervous system dysregulation of the modulation of gut signaling and motor function [1]. FGIDs lack a structural basis to explain their clinical features: the general scientific understanding of these disorders adheres to a biopsychosocial model [3]. Although these different diseases affect different regions of the digestive tract, it has been noted that they seem to occur frequently in the same patient. Irritable bowel syndrome (IBS) and functional dyspepsia (FD) are considered the most frequent FGIDs. 
Gastroesophageal reflux disease (GERD) is an upper gastrointestinal disorder that affects a large part of the general population, with a relevant and significant impact on quality of life and healthcare costs. GERD consists of troublesome symptoms or mucosal damage due to the retrograde movement of gastric content through an incompetent esophagogastric junction [4]. GERD is one of the most common gastrointestinal ailments worldwide; up to $40 \%$ of the US population report esophageal symptoms intermittently and 10-20\% have at least weekly symptoms [5]. Typical GERD symptoms consist of heartburn and regurgitation, and clinical diagnosis is made on the basis of typical symptoms, supported by the response of symptoms to empiric proton pump inhibitor (PPI) therapy [6,7].

Over the past decade, it has been realized that the majority of GERD patients (approximately 70\%) have typical reflux symptoms (i.e., heartburn, regurgitation) without any esophageal mucosal breaks on upper endoscopy; thus, they are considered to have non-erosive reflux disease (NERD) [4,8-10]. Consistently with this definition, a GERD diagnosis can be suspected based on the presence of typical symptoms only. However, several recent studies have underlined that NERD represents a heterogeneous group of patients with several pathophysiological and clinical differences and should be better classified using appropriate techniques able to characterize gastroesophageal refluxate, because both the management and the therapeutic responses change depending on the main mechanism of symptom generation [11-15].

Multichannel intraluminal impedance and $\mathrm{pH}$ monitoring (MII-pH) is considered the gold standard to identify GERD patients, and distal esophageal acid exposure time (AET), the number of reflux episodes and the association between symptoms and refluxes can also be evaluated [16-19]. Recently, up-and-coming parameters, such as baseline impedance values [20-22] and post-reflux swallowing-induced peristaltic wave index [23-25], have been described to better distinguish patients with GERD from those without.

The overlap between IBS, FD and GERD has been widely reported in the literature [26-29]. The majority of studies evaluating the overlap of FGIDs have focused on only one or two FGID combinations, most notably IBS and FD. A relevant issue is to understand the degree of overlap between these conditions and whether they share any pathophysiological mechanism that can substantiate their association.

The aim of this review was to show and discuss, based on our current best understanding, the overlap between GERD, with special attention to functional heartburn $(\mathrm{FH})$, and various gastrointestinal functional disorders, such as IBS and FD.

\section{GERD and FH}

Typical symptoms of GERD, such as heartburn and regurgitation, are widespread in the community. The prevalence of GERD based on symptom perception in individual cross-sectional surveys varies from $2.5 \%$ to more than $25 \%[5,30]$. GERD occurs less frequently in Asia than in Western countries [31,32].

The worldwide prevalence of GERD has increased during the past 15 years. GERD may be classified into two different forms: erosive reflux disease (ERD) and NERD; the latter represents the majority of reflux patients (up to 70\%), who have typical symptoms without any esophageal mucosal lesion visible on endoscopy [33]. The severity of reflux esophagitis is usually classified according to the Los Angeles criteria (from A to D denoting increasing severity and extension of inflammation) [34].

The diagnosis of NERD should be supported by evidence that symptoms are due to acid reflux, on the basis of an excess of acid, or a positive correlation between symptoms and acid reflux using functional $\mathrm{pH}$ testing, or evidence of a symptomatic response to acid suppression [35,36]. Moreover, the use of 24-h MII-pH has shown that weak acidic reflux is also able to induce typical reflux symptoms, which, however, do not respond to PPIs [37-39].

Recently, the Rome IV criteria for functional esophageal disorders defined $\mathrm{FH}$ as retrosternal burning discomfort or pain refractory to optimal antisecretory therapy in the absence of GERD (i.e., normal acid exposure and no evidence of a symptom-reflux correlation), histopathologic mucosal abnormalities, major motor disorders or organic disease [40]. Notably, Savarino et al [16] suggested that, to be diagnosed with $\mathrm{FH}$, patients should display a normal upper endoscopy, a normal AET in the distal esophagus and a negative symptom association with both acid and non-acid reflux by means of MII-pH.

\section{IBS}

IBS is a functional intestinal disorder characterized by recurrent abdominal pain, on average at least 1 day per week over a 3-month period, associated with two or more of the following criteria: the pain is related to defecation; the pain is associated with a change in frequency of stool and/or with a change in form (appearance) of stool [41]. IBS has traditionally been subcategorized into four subtypes based on predominant stool pattern: with constipation (IBS-C); with diarrhea (IBS-D); mixed (IBS-M) or unsubtyped (IBS-U) [42].

An estimated 5-25\% of the people worldwide suffer from IBS. It is now recognized as the most common chronic bowel disorder $[42,43]$. Overlap of GERD and IBS symptoms ranges between $5 \%$ and $30 \%$ in community individuals $[26,44,45]$, whereas the underlying mechanisms for this overlap and the cardinal symptoms and characteristics of GERD patients with IBS remain unclear. A detailed identification of prevalent symptoms is determinant of GERD.

\section{FD}

According to Rome IV criteria, the broad term FD comprises patients from the diagnostic categories of postprandial distress syndrome (PDS), characterized by meal-induced dyspeptic symptoms; epigastric pain syndrome (EPS), which refers to epigastric pain or epigastric burning that does not occur exclusively postprandially, can occur during fasting, and can even be improved by meal ingestion; and overlapping PDS and EPS, characterized by meal-induced dyspeptic symptoms and epigastric pain or burning. Moreover, the diagnostic criteria 
for FD specify that there should be no evidence of structural disease likely to explain the symptoms: i.e., upper endoscopy is normal [46]. Large-scale studies reported a $10-30 \%$ prevalence of FD worldwide, depending on the definition used and the geographical location [47].

\section{IBS and GERD overlap: questionnaire-based diagnosis}

In a recent review article, our group appraised and critically discussed studies supporting a possible overlap of GERD and FH with IBS. Notably, in studies where heartburn was investigated through only a symptom questionnaire, we found remarkable variability regarding the prevalence of GERD in patients with a diagnosis of IBS (Manning, Rome I, Rome II, Rome III criteria), with values ranging from 3-79\% [48]. In 2012, Ford et al [45] conducted a systematic review and meta-analysis to estimate the prevalence of GERD-type symptoms in individuals with IBS and to quantify the overlap between the disorders. The authors selected population-based studies reporting the prevalence of IBS and GERD-type symptoms defined using symptom-based criteria or questionnaires. Overall, they selected 13 studies, in which the prevalence of GERD-type symptoms in IBS was $42 \%$; the overall odds ratios of GERD-type symptoms in individuals with IBS was fourfold that of individuals without IBS. In particular, the positive association between GERDtype symptoms and IBS persisted in all geographical regions examined, and was consistent with each definition of IBS and gastroesophageal reflux symptoms, although the degree of overlap varied between $12 \%$ and $27 \%$, depending on the diagnostic criteria used to define each condition [45].

In addition to the aforementioned meta-analysis, an Arab cross-sectional survey study found that $74.7 \%$ of IBS patients (198 individuals, Rome III criteria) had GERD symptoms at the same time (i.e., heartburn and/or acid regurgitation at least once per week for the last 3 months) [49]. More recently, Rasmussen et al [50] investigated the prevalence and overlap of these two conditions in a general Western population. In this nationwide study, 49,706 randomly selected individuals were eligible and underwent a web-based questionnaire survey, which showed the prevalences of GERD (Montreal definition) and IBS (Rome III criteria) to be $11.2 \%$ and $10.5 \%$, respectively. Notably, the overlap between GERD and IBS was $56.9 \%$.

\section{IBS and GERD overlap: endoscopic diagnosis}

Few data are available regarding the prevalence of IBS in patients with erosive esophagitis. The overall prevalence of IBS symptoms in the GERD population ranges from $10-74 \%$, and it seems to be more frequent in patients with NERD than those with ERD. We focused our attention on some populationbased studies in which GERD was detected using endoscopy. Few studies have reported pathophysiological evaluations to exclude patients with FH from the NERD population.

First, Smart et al [51] described an overlap with IBS symptoms (Manning criteria) in 9/22 (40.9\%) patients with erosive esophagitis and/or esophageal complications. Neumann and coworkers [52] evaluated the prevalence of IBS symptoms (Rome III criteria) in patients with GERD classified by subgroup into NERD, ERD and Barrett's esophagus (BE). They observed that IBS was common in the entire spectrum of GERD: $63 \%$ in NERD, $44 \%$ in ERD and $21 \%$ in BE patients. The authors concluded that the presence of these disorders might explain why many patients with GERD have no complete symptom relief with PPI treatment. In 118 Mexican patients with IBS (Rome I criteria), Camacho et al [53] showed a general prevalence of IBS symptoms (48\%) in patients with ERD and NERD. In a group of ERD (286) and NERD (74) patients from South Korea, Noh et al [54] described a prevalence of IBS (Rome III criteria) of $10.1 \%$. However, the proportion of IBS-related symptoms was higher in NERD than in ERD patients $(74.3 \%$ vs. $10.5 \%)$. In a different and more recent Korean study by Nam et al [55], who aimed to evaluate the association among IBS (Rome III criteria), NERD and ERD, a total of 2769 participants completed questionnaires and underwent upper and lower endoscopy. Psychological distress was also evaluated and was associated with both IBS and NERD, but not with ERD. Overall, the authors concluded that IBS was positively associated with NERD, but found no association with ERD [55].

In a case-control study from Taiwan, Hsu et al [56] evaluated the prevalence of IBS (Rome III criteria) in patients with and without esophageal-related symptoms (including ERD and NERD patients). The prevalence of IBS was at least $10 \%$ in patients with GERD and $5.5 \%$ in those without. The authors found that patients with GERD and IBS reported more frequent healthcare-seeking behavior, more severe GERD symptoms, poorer sleep quality, and greater depression than did those with IBS or GERD alone [56].

Monnikes et al [57] evaluated and treated a large number of patients with ERD (626 intention-to-treat; 500 per protocol analysis) with pantoprazole (up to 16 weeks). They described an overlap with IBS symptoms (Rome II/III criteria) in 13.6\% of patients. At the end of the treatment period, they observed that all patients showed healed esophageal erosions but, more interestingly, that only $4.7 \%$ continued to report IBS symptoms. This percentage decreased to $2.8 \%$ after 6 months. They concluded that pantoprazole was effective in the treatment of patients suffering from signs and symptoms that suggested an overlap of GERD and IBS by providing a sustained response in the posttreatment period. After adjusting for many confounding factors, including psychological stress and Helicobacter pylori infection, Namm et al showed that IBS symptoms had a strong positive association with NERD, but no association with ERD [55].

\section{IBS and GERD overlap: pathophysiological examination}

The diagnosis of IBS is clinically based on the presence of characteristic symptoms, such as abdominal pain or discomfort and altered bowel habits. Lower endoscopy or hematological examinations are indicated only to exclude organic diseases, but these tests are not compulsory to correctly diagnose IBS [42]. Likewise, a GERD diagnosis could be clinically suspected and 
characterized by the presence of "troublesome" heartburn or regurgitation [4].

Within the spectrum of GERD, the pathophysiological relationship between ERD and NERD remains a subject of debate [58]. The classical and perhaps intuitive view, in which NERD is a mild form of GERD that might progress with time to ERD, is supported by some physiological, anatomical and histopathological findings [59]. However, NERD is a large umbrella that includes patients with reflux symptoms and negative upper endoscopy and this diagnosis is often made without any effort to distinguish FH (defined by the Rome III criteria) from NERD using pathophysiological investigations [60].

Currently, $\mathrm{FH}$ is a disorder characterized by symptoms of heartburn not related to GERD, diagnosed using 24-h impedance-pH testing, and it must be distinguished from NERD [16,33,61-63]. We previously described that the prevalence of GERD in the IBS population was $37.5 \%$, with a large variability ranging from 11-79\% [48]. First, Smart et al described abnormal reflux parameters using $\mathrm{pH}$-metry in $11(50 \%)$ of 22 patients with an IBS diagnosis (Manning criteria) [51]. Hershcovici et al [64] studied a high prevalence (49\%) of IBS-related symptoms in a large cohort of patients with NERD (defined as abnormal 24-h pH-metry). Zimmerman et al described 256 patients with GERD, 50\% of whom met the criteria for IBS. The authors concluded that in patients with NERD, the relationship between AET and symptoms was affected significantly, and in opposite directions, by smoking and IBS [65].

Using 24-h MII-pH, Martinucci et al [66] observed that IBS overlaps more frequently with $\mathrm{FH}(66 \%)$ than with ERD (17\%) or NERD (39.5\%). Recently, Garros et al [67] evaluated 168 patients with GERD symptoms classified into subgroups of responders (26) and non-responders (126) to PPI treatment. All patients underwent pathophysiological examinations. The authors concluded that IBS was more frequent in patients who did not respond to PPI treatment $(27.2 \%)$ than in the responder group (7.7\%). However, the authors did not report the prevalence of various subcategories of GERD in patients who showed the overlap with functional symptoms.

A recent meta-analysis showed that the odds ratio for GERD-related symptoms in individuals with IBS was fourfold that of individuals without IBS [45]. Accordingly, we can speculate that patients with GERD symptoms include subjects with either abnormal (NERD) or normal acid exposure, as well as FH patients. Savarino et al [33] found a greater prevalence of dyspeptic symptoms in patients with $\mathrm{FH}$ than in patients with NERD. The authors suggested that the data reinforce the concept that FGIDs extend beyond the boundaries suggested by the anatomical location of symptoms. This conclusion should be considered a further argument for testing patients with symptoms of GERD to separate patients with FH from patients with NERD [33]. In Fig. 1 we report the estimated overlap between GERD and IBS according to the Rome III and Rome IV definitions. More in-depth pathophysiological examinations are needed to detect patients with NERD and those with FH to better estimate the overlap between patients with heartburn and IBS.

\section{FD and GERD overlap: questionnaire-based diagnosis}

In 2011, a systematic literature review found that dyspeptic symptoms of epigastric pain, early satiety, bloating, nausea and vomiting were more common in patients with frequent GERD symptoms (range 21-63\%) than in those with intermittent or no GERD symptoms [68]. A Korean population-based study, using the Rome III criteria, found an overlap between GERD and uninvestigated dyspepsia in 50\% of GERD patients [69]. However, it should be noted that uninvestigated dyspepsia includes patients with an organic, systemic, or metabolic cause for the symptoms that could have been identified by traditional diagnostic procedures, which were not carried out.

A nationwide study of 100,000 individuals investigated the prevalence and overlap of GERD, FD and IBS in a Western general population by means of a web-based questionnaire survey. Among individuals fulfilling the criteria for GERD, FD and IBS, $69.3 \%$ fulfilled the criteria for only one of the conditions, $22.9 \%$ for two, while $7.7 \%$ fulfilled the criteria for all three. Among individuals meeting the criteria for one or more of the conditions, $30.7 \%$ fulfilled the criteria for two or all three conditions [28].

Interestingly, a study by Pleyer et al [70] found evidence supporting a systematic bias away from diagnosing FD, favoring a GERD diagnosis. Indeed, the authors assessed survey-based upper gastrointestinal symptom reporting between 1988 and 2009 and, though the majority of subjects (63\%) reporting GERD symptoms received a GERD diagnosis, only a minority (13\%) of those reporting FD symptoms received an appropriate FD diagnosis [70]. Such observer bias may be an important factor that hinders a correct assessment of the prevalence and overlap between these syndromes.

\section{FD and GERD overlap: endoscopic diagnosis}

In a study of 171 GERD patients, cases with symptoms compatible with FD, based on the Rome III diagnostic criteria and negative endoscopic findings, were assigned to the overlapping GERD-FD group (28\% of enrolled patients). Patients with concomitant GERD and FD were more likely

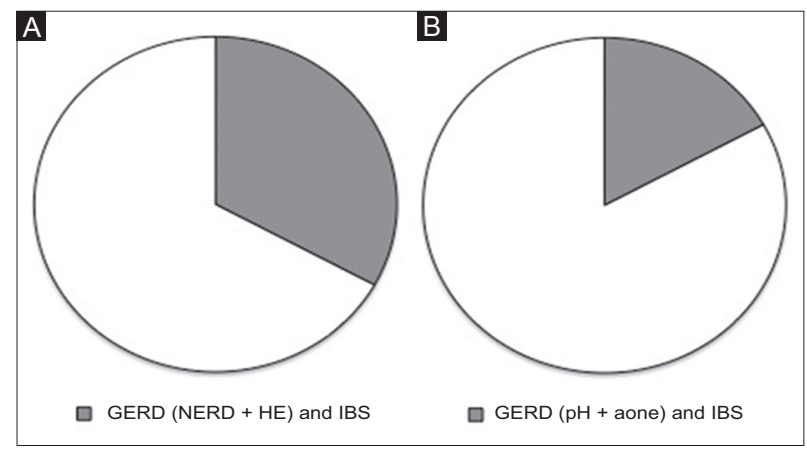

Figure 1 (A) Different overlap between GERD and IBS according to Rome III (B) and Rome IV GERD, gastroesophageal reflux disease; NERD, non-erosive reflux disease; HE, hypersensitive esophagus; IBS, irritable bowel syndrome 
to be younger and female. Moreover, overlapping GERD and FD had the worst impact on the quality of life of the affected individuals, compared with GERD only and overlapping GERD and peptic ulcer disease [71].

\section{FD and GERD overlap: pathophysiological examination}

Abnormal $\mathrm{pH}$ monitoring was found in $23 \%$ of 247 patients with FD. [72] Subsequently, in a study by Savarino et al [33], 24-h ambulatory MII-pH data and FD questionnaires collected prospectively from a large group of NERD patients showed that clinically relevant dyspeptic symptoms were present in $44 \%$ of the NERD population. In particular, this study showed that when NERD patients were classified into three different subgroups on the basis of MII-pH results, dyspeptic symptoms were reported more frequently in patients with $\mathrm{FH}(63 \%)$, compared to those who had NERD with abnormal acid exposure time (41\%) or hypersensitive esophagus (37\%), suggesting a significantly different degree of overlap of NERD subgroups with FD [33]. Fig. 2 presents the estimated overlap between GERD and FD according to the Rome III and Rome IV definitions.

\section{Pathophysiological similarities in GERD, FD and IBS}

From a pathophysiological point of view, FGIDs are defined as gut-brain interaction disorders, characterized by visceral hypersensitivity, motility disturbance, altered mucosal and immune function, altered gut microbiota and altered central nervous system processing [41]. Notably, previous studies suggested that visceral hypersensitivity, minimal abnormalities of motility, and peripheral and central neural mechanisms could be considered the main common pathophysiological mechanisms among GERD and FGIDs [73,74].

It has been shown that patients with FGIDs exhibit a reduced pain/discomfort threshold during visceral stimulation [75]. Visceralhypersensitivity tovariousstimuli(chemical,distension, physical stimuli) has been demonstrated in patients with both IBS-related [76] and GERD-related [77] symptoms. Costantini et al [78] performed esophageal provocative testing (balloon distension and bethanechol) in IBS patients and healthy controls. They observed that esophageal symptoms appeared more frequently in patients with IBS, without any evidences of abnormal esophageal motility. Likewise, FD patients are also sensitive to acid, whether mediated through gastric acid "sensitivity" or impaired duodenal acid clearance $[79,80]$.

Motor abnormalities are frequently reported as a common pathophysiological mechanism in both GERD and IBS [81]. Jones et al [82] speculated that the overlap between IBS and GERD could be explained by an unspecified dysfunction of smooth muscle across the gastrointestinal tract. Motor dysfunction of the upper stomach has also been described in patients with overlapping GERD and FD. Gonlachanvit et al [83] observed that gastric emptying was delayed in about $50 \%$ of patients with GERD, FD, and overlapping GERD and FD; proximal retention was greatest in patients with GERD and
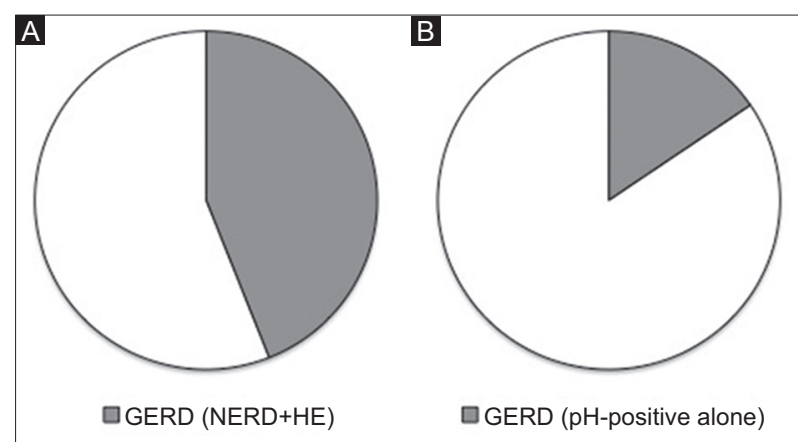

Figure 2 (A) Different overlap between GERD and FD according to Rome III (B) and Rome IV GERD, gastroesophageal reflux disease; NERD, non-erosive reflux disease; HE, hypersensitive esophagus; FD, functional dyspepsia

distal retention was a feature of both FD and the overlap groups. Moreover, van Lelyveld et al [84] showed that proximal gastric volume was smaller in FD and larger in GERD than in healthy control patients.

Hsu et al showed that GERD patients with more severe GERD symptoms, poor sleep quality, and greater depression are at higher risk of comorbid IBS. Likewise, IBS patients who reported poor sleep quality had a higher risk for GERDrelated symptoms. The authors speculated that behavioral and psychological symptoms such as sleep disturbance and depression could be considered as risk factors to explain the overlap between GERD and IBS [85].

Psychosocial factors may also predispose individuals to the development of IBS [86]. Rubenstein et al [87] observed that patients with heartburn who reported increased levels of psychological distress and IBS-related symptoms recorded abnormal esophageal perception of both acid perfusion and balloon distension.

\section{Effect of GERD treatment on FD and IBS}

The majority of GERD patients are directly managed by general practitioners and receive PPI empirically, based on the fact that PPIs have a very low toxicity profile and good efficacy for suppressing gastric acid secretion and symptom relief. This approach is named "PPI trial" and it commonly involves a high dose of PPIs for at least 4 weeks [88]. A major limitation to note is its moderate sensitivity and specificity ( $78 \%$ and $54 \%$, respectively) as a test to discriminate refluxrelated manifestations [89]. However, the safety, efficacy and relatively low cost of these medications justify the use of PPIs as a first tool in a "step-down" approach for patients with GERD symptoms. Nevertheless, a "step-up" strategy is commonly adopted in patients with mild and intermittent symptoms, with the therapy strengthened every 2-4 weeks in case of lack of benefit. Regardless of the strategy undertaken, the management of GERD must include the avoidance of risk factors through lifestyle and dietary modifications $[88,90]$. Subsequently, when medical therapies are considered, acid secretion suppressors are considered the most effective in determining adequate 
symptom relief. There are also some reports that suggest an improvement in GERD-related symptoms after treatment with alginates [91,92].

Althoughtherehasbeenconsistent progressinunderstanding the pathophysiology of GERD $[11,21,23,38,62,93]$ and the several therapeutic options available, a considerable proportion of patients do not achieve a satisfactory therapeutic benefit. Therefore, the management of refractory GERD remains a disorder to be treated in specialized settings.

PPI therapy is undoubtedly associated with a superior healing rate and symptom control, as well as a lower rate of relapse compared with placebo, histamine-2 receptor antagonists, sucralfate, and prokinetics [94-97]. Although the acid content of the refluxate plays the main pathogenetic role in GERD, there are several factors that contribute to the disease, including alkaline reflux, pepsin, visceral hypersensitivity and psychological stress. The presence of concomitant functional disorders (IBS and FD) is, indeed, strictly related to a minor response to medical treatment. In fact, functional disorders, especially IBS, are more frequent in NERD patients than in ERD $[33,66,98]$. Translated into clinical terms, PPIs have greater benefit in ERD (70-80\%) patients than in those with NERD (50-60\%) [99-101]. However, the response rates vary according to the criteria employed to classify patients, the dosage administered and the duration of therapy. In fact, better discrimination among functional disorders, higher dosage and prolonged therapy are associated with a higher response rate to the treatment. The influence of functional disorders on treatment response seems to have been confirmed by Zerbib et al [102], who showed that the absence of esophagitis, the presence of FGIDs and a body mass index $\leq 25$ are strongly associated with PPI failure in patients with GERD-related symptoms. Likewise, Garros et al [67] showed that the percentage of patients with FD and IBS was higher in non-responders to PPI than in responders $(65.6 \%$ and $27.2 \%$, respectively, vs. $38.5 \%$ and $7.7 \%)$. In this regard, Mönnikes et al showed that the presence of IBS-like symptoms reduced the response to PPIs in GERD patients, regardless of whether they had ERD or NERD [103].

It is worth noting that NERD is a term that includes three different types of patients characterized by various responses to the medical therapy: "true NERD", hypersensitive esophagus and $\mathrm{FH}$ [12]. As proven in the meta-analysis by Weijenborg, if patients are diagnosed with NERD based on MII-pH (true NERD), the response to PPI treatment is as successful as in those affected by ERD [104]. In patients with NERD, there is no evidence to recommend maintenance treatment, even though approximately $70-75 \%$ of NERD patients relapse off therapy $[105,106]$. In this regard, some authors have suggested that an "on-demand" PPI treatment may be an alternative option $[107,108]$. Wu et al [109] showed, in a group of GERD patients (163 NERD and 102 ERD), that NERD patients have a higher failure rate of on-demand PPI therapy than do patients with ERD, especially if the former reported concomitant IBS symptoms.

A different subcategory of GERD that would benefit from PPI treatment includes patients with normal AET but a positive symptom-acid reflux relation (i.e., hypersensitive esophagus) [110]. The Rome IV consensus reconsidered patients with hypersensitive esophagus as functional patients.

Greater difficulties are encountered with NERD patients who do not have excessive AET and any relationship between symptoms and acid or weakly acidic refluxes. This subgroup of patients with $\mathrm{FH}$ is no longer classified under the term of GERD. Since these patients are typically poor responders to PPIs $[35,110]$, other drugs aimed at reducing visceral sensitivity should be considered. To date, the medications that have been better investigated are tricyclic antidepressant agents, selective serotonin reuptake inhibitors and serotoninnorepinephrine reuptake inhibitors. Unfortunately, there are few placebo-controlled trials aimed at demonstrating the effectiveness of such compounds. Evidence from studies conducted on patients with IBS and functional dyspepsia have shown that there might be an overlap between FH and IBS [61,111,112]. Moreover, recent data obtained by Viazis et al [113] seem to support this argument. Their study demonstrated that citalopram, a selective serotonin reuptake inhibitor, provides greater benefit than placebo in controlling heartburn in hypersensitive patients. In contrast to $\mathrm{FH}$, HE patients belong to the GERD population. However, IBS symptoms are often coexistent and the efficacy of PPI therapy is lower than in NERD and EE patients.

The relationship between IBS and GERD is under debate. One hypothesis suggests that IBS and GERD are two different pathologies that possess overlapping pathophysiologic processes $[114,115]$. Therefore, patients should receive two distinct treatments, each of which may affect the symptoms related to the other condition only minimally or not at all. Another hypothesis proposes that IBS is an extra-esophageal manifestation of GERD $[116,117]$. Several trials support this argument, reporting IBS symptom resolution in $20-40 \%$ of patients after PPI therapy $[116,118]$. Likewise, in a large-scale clinical study, lansoprazole effectively relieved dyspepsia in addition to reflux symptoms in patients with GERD [119]. Recently, another study showed that, after 4 weeks of treatment with lansoprazole, $>60 \%$ of both patients with NERD and FD reported an improvement in reflux symptoms and in most dyspepsia symptoms [120]. In line with these results, Mönnikes et al observed improvement of FD and IBS symptoms after PPI therapy in GERD patients [57]. Surprisingly, there was no recrudescence of symptomatology after the treatment was suspended, and a sustained response with pantoprazole $40 \mathrm{mg}$ was maintained up to 16 weeks after treatment [57].

Nevertheless, it should be kept in mind when prescribing PPIs that these medications might be directly involved in the genesis, or may at least favor ("two-hit" hypothesis) the onset, of IBS. Indeed, various mechanisms have been hypothesized: alteration in intestinal permeability $[121,122]$ and alteration of the intestinal barrier function [123] and the composition of intestinal microbiota [124], which in turn may induce secondary changes in epithelial integrity [125].

Recently, a long period of treatment with PPIs was associated with increased risk of developing microscopic colitis [126], while one investigator speculated about a possible association between microscopic colitis and changes in intestinal permeability [121]. 
PPIs have been associated with some described alterations in intestinal barrier function [123] and subsequently with secondary important changes in intestinal microbiota composition [124]. It might be possible to speculate that these changes play a determining role in IBS pathogenesis.

Currently, scant data are available on the complex topic of overlapping FGIDs. MII-pH certainly contributes to tailoring reflux treatment by discriminating among different types of patients in the NERD realm, characterized by various responses to treatment $[12,33,63]$. Furthermore, a recent study showed that MII-pH could potentially be a helpful tool for predicting the response to PPIs in patients with heartburn [110]. However, further case-control trials, including studies with a placebo arm and conducted with accurate identification of upper and lower FGIDs, are needed.

\section{Therapeutic options in IBS}

The goal of IBS treatment is to provide relief of symptoms and improve overall quality of life. Because the symptoms of IBS are so wide-ranging, each patient must receive therapy individualized to achieve these goals. Currently, the ideal pharmacological therapy in patients with IBS would be one that completely addresses the entire IBS symptom complex, with minimal or no adverse side effects. Medications that modulate visceral hypersensitivity should do so without adversely affecting cognitive functions, somatic perception or gastrointestinal motility [127].

In the largest study to date of selective serotonin reuptake inhibitor therapy for IBS, 257 patients with severe IBS were randomized to paroxetine $(20 \mathrm{mg} / \mathrm{day})$, psychotherapy or usual care. After 12 weeks of treatment, the paroxetine group experienced a small but significant reduction in the number of days with abdominal pain compared to baseline $(\mathrm{P}=0.014)$ as well as improvements in their quality of life [128]. Another recent cross-over trial found that citalopram was more effective than placebo in improving abdominal pain, bloating and overall wellbeing in 23 non-depressed IBS patients referred to a tertiary care center in Belgium [129]. The mechanisms via which antidepressants have a beneficial effect on IBS are not clear. It is possible that these effects may reflect an improvement in the psychiatric profile of these patients, as many have anxiety and depression as a comorbidity.

\section{Concluding remarks}

Our current knowledge about the overlap of "true" GERD and IBS, as well as of GERD and FD, is limited. A large number of studies aimed to detect these overlaps, but only a few were performed according to pathophysiologic criteria. Obviously, the recent modification of the definitions of functional esophageal disorders in the Rome IV criteria may alter the prevalence and overlap between GERD and other functional diseases of the gut. Over the last 10 years, few studies have been conducted to better understand the pathophysiological origin of heartburn in IBS or FD patients. The more recent pathophysiological studies showed that functional esophageal disorders overlap more frequently with IBS or FD compared with GERD. A deeper comprehension of the pathophysiology should improve therapeutic options in the treatment of FGIDs.

\section{References}

1. Drossman DA, Hasler WL. Rome IV-Functional GI Disorders: disorders of gut-brain interaction. Gastroenterology 2016;150:1257-1261.

2. Koloski NA, Talley NJ, Boyce PM. Epidemiology and health care seeking in the functional GI disorders: a population-based study. Am J Gastroenterol 2002;97:2290-2299.

3. Drossman DA. Presidential address: Gastrointestinal illness and the biopsychosocial model. Psychosom Med 1998;60:258-267.

4. Vakil N, van Zanten SV, Kahrilas P, Dent J, Jones R; Global Consensus Group. The Montreal definition and classification of gastroesophageal reflux disease: a global evidence-based consensus. Am J Gastroenterol 2006;101:1900-1920.

5. El-Serag HB, Sweet S, Winchester CC, Dent J. Update on the epidemiology of gastro-oesophageal reflux disease: a systematic review. Gut 2014;63:871-880.

6. Katz PO, Gerson LB, Vela MF. Guidelines for the diagnosis and management of gastroesophageal reflux disease. Am J Gastroenterol 2013;108:308-328.

7. Savarino E, Bredenoord AJ, Fox M, Pandolfino JE, Roman S, Gyawali CP; International Working Group for Disorders of Gastrointestinal Motility and Function. Expert consensus document: advances in the physiological assessment and diagnosis of GERD. Nat Rev Gastroenterol Hepatol 2017;14:665-76.

8. Modlin IM, Hunt RH, Malfertheiner P, et al; Vevey NERD Consensus Group. Diagnosis and management of non-erosive reflux disease-the Vevey NERD Consensus Group. Digestion 2009;80:74-88.

9. Savarino E, Marabotto E, Bodini G, et al. Epidemiology and natural history of gastroesophageal reflux disease. Minerva Gastroenterol Dietol 2017;63:175-183.

10. Savarino E, de Bortoli N, De Cassan C, et al. The natural history of gastro-esophageal reflux disease: a comprehensive review. Dis Esophagus 2017;30:1-9.

11. de Bortoli N, Martinucci I, Savarino E, et al. Proton pump inhibitor responders who are not confirmed as GERD patients with impedance and $\mathrm{pH}$ monitoring: who are they? Neurogastroenterol Motil 2014;26:28-35.

12. Savarino E, Zentilin P, Savarino V. NERD: an umbrella term including heterogeneous subpopulations. Nat Rev Gastroenterol Hepatol 2013;10:371-380.

13. Giacchino M, Savarino V, Savarino E. Distinction between patients with non-erosive reflux disease and functional heartburn. Ann Gastroenterol 2013;26:283-289.

14. Tolone S, De Bortoli N, Marabotto E, et al. Esophagogastric junction contractility for clinical assessment in patients with GERD: a real added value? Neurogastroenterol Motil 2015;27:1423-1431.

15. Frazzoni L, Frazzoni M, de Bortoli N, et al. Postreflux swallowinduced peristaltic wave index and nocturnal baseline impedance can link PPI-responsive heartburn to reflux better than acid exposure time. Neurogastroenterol Motil 2017;29.

16. Savarino E, Zentilin P, Tutuian R, et al. The role of nonacid reflux in NERD: lessons learned from impedance-pH monitoring in 
150 patients off therapy. Am J Gastroenterol 2008;103:2685-2693.

17. Bredenoord AJ. Impedance-pH monitoring: new standard for measuring gastro-oesophageal reflux. Neurogastroenterol Motil 2008;20:434-439.

18. Sifrim D. Acid, weakly acidic and non-acid gastro-oesophageal reflux: differences, prevalence and clinical relevance. Eur $J$ Gastroenterol Hepatol 2004; 16:823-830.

19. Tolone S, de Cassan C, de Bortoli N, et al. Esophagogastric junction morphology is associated with a positive impedance$\mathrm{pH}$ monitoring in patients with GERD. Neurogastroenterol Motil 2015;27:1175-1182.

20. Kessing BF, Bredenoord AJ, Weijenborg PW, Hemmink GJ, Loots CM, Smout AJ. Esophageal acid exposure decreases intraluminal baseline impedance levels. Am J Gastroenterol 2011;106:2093-2097.

21. Martinucci I, de Bortoli N, Savarino E, et al. Esophageal baseline impedance levels in patients with pathophysiological characteristics of functional heartburn. Neurogastroenterol Motil 2014;26:546-555.

22. Frazzoni M, Savarino E, de Bortoli N, et al. Analyses of the postreflux swallow-induced peristaltic wave index and nocturnal baseline impedance parameters increase the diagnostic yield of patients with reflux disease. Clin Gastroenterol Hepatol 2016;14:40-46.

23. Frazzoni M, Manta R, Mirante VG, Conigliaro R, Frazzoni L, Melotti G. Esophageal chemical clearance is impaired in gastroesophageal reflux disease-a 24-h impedance- $\mathrm{pH}$ monitoring assessment. Neurogastroenterol Motil 2013;25:399-406.

24. Frazzoni M, de Bortoli N, Frazzoni L, Tolone S, Savarino V, Savarino E. Impedance-pH monitoring for diagnosis of reflux disease: new perspectives. Dig Dis Sci 2017;62:1881-1889.

25. Tenca A, de Bortoli N, Mauro A, et al. Esophageal chemical clearance and baseline impedance values in patients with chronic autoimmune atrophic gastritis and gastro-esophageal reflux disease. Dig Liver Dis 2017;49:978-983.

26. Kennedy TM, Jones RH, Hungin AP, O'flanagan H, Kelly P. Irritable bowel syndrome, gastro-oesophageal reflux, and bronchial hyper-responsiveness in the general population. Gut 1998;43:770-774.

27. Jung HK, Halder S, McNally $M$, et al. Overlap of gastrooesophageal reflux disease and irritable bowel syndrome: prevalence and risk factors in the general population. Aliment Pharmacol Ther 2007;26:453-461.

28. Quigley EM, Lacy BE. Overlap of functional dyspepsia and GERD - diagnostic and treatment implications. Nat Rev Gastroenterol Hepatol 2013;10:175-186.

29. Choung RS, Locke GR $3^{\text {rd }}$, Schleck CD, Zinsmeister AR, Talley NJ. Overlap of dyspepsia and gastroesophageal reflux in the general population: one disease or distinct entities? Neurogastroenterol Motil 2012;24:229-234.

30. Cho YS, Choi MG, Jeong JJ, et al. Prevalence and clinical spectrum of gastroesophageal reflux: a population-based study in Asan-si, Korea. Am J Gastroenterol 2005;100:747-753.

31. Dent J, El-Serag HB, Wallander MA, Johansson S. Epidemiology of gastro-oesophageal reflux disease: a systematic review. Gut 2005;54:710-717.

32. Mahadeva S, Raman MC, Ford AC, et al. Gastro-oesophageal reflux is more prevalent in Western dyspeptics: a prospective comparison of British and South-East Asian patients with dyspepsia. Aliment Pharmacol Ther 2005;21:1483-1490.

33. Savarino E, Pohl D, Zentilin P, et al. Functional heartburn has more in common with functional dyspepsia than with nonerosive reflux disease. Gut 2009;58:1185-1191.

34. Lundell LR, Dent J, Bennett JR, et al. Endoscopic assessment of oesophagitis: clinical and functional correlates and further validation of the Los Angeles classification. Gut 1999;45:172-180.
35. Savarino E, Zentilin P, Martinato M, Savarino V. Nonerosive reflux disease and functional heartburn are clearly separate entities. Eur J Gastroenterol Hepatol 2013;25:749-750.

36. Gyawali CP, Kahrilas PJ, Savarino E, et al. Modern diagnosis of GERD: the Lyon Consensus Gut 2018;67:1351-1362.

37. de Bortoli N, Martinucci I, Savarino EV, et al. Manually calculated oesophageal bolus clearance time increases in parallel with reflux severity at impedance-pH monitoring. Dig Liv Dis 2015;47:1027-1032.

38. de Bortoli N, Martinucci I, Savarino E, et al. Lower pH values of weakly acidic refluxes as determinants of heartburn perception in gastroesophageal reflux disease patients with normal esophageal acid exposure. Dis Esophagus 2016;29:3-9.

39. de Bortoli N, Ottonello A, Zerbib F, Sifrim D, Gyawali CP, Savarino E. Between GERD and NERD: the relevance of weakly acidic reflux. Ann N Y Acad Sci 2016;1380:218-229.

40. Aziz Q, Fass R, Gyawali CP, Miwa H, Pandolfino JE, Zerbib F. Functional esophageal disorders. Gastroenterology 2016;150:1368-1379.

41. Drossman DA. Functional gastrointestinal disorders: history, pathophysiology, clinical features and Rome IV. Gastroenterology 2016;150:1262-1279.e2.

42. Drossman DA. The functional gastrointestinal disorders and the Rome III process. Gastroenterology 2006;130:1377-1390.

43. Khoshkrood-Mansoori B, Pourhoseingholi MA, Safaee A, et al. Irritable bowel syndrome: a population based study. J Gastrointestin Liver Dis 2009;18:413-418.

44. Ford AC, Forman D, Bailey AG, Axon AT, Moayyedi P. Fluctuation of gastrointestinal symptoms in the community: a 10-year longitudinal follow-up study. Aliment Pharmacol Ther 2008;28:1013-1020.

45. Lovell RM, Ford AC. Prevalence of gastro-esophageal refluxtype symptoms in individuals with irritable bowel syndrome in the community: a meta-analysi. Am J Gastroenterol 2012;107:1793-1801.

46. Stanghellini V, Chan FK, Hasler WL, et al. Gastroduodenal disorders. Gastroenterology 2016;150:1380-1392.

47. Mahadeva S, Goh KL. Epidemiology of functional dyspepsia: a global perspective. World J Gastroenterol 2006;12:2661-2666.

48. de Bortoli N, Martinucci I, Bellini M, et al. Overlap of functional heartburn and gastroesophageal reflux disease with irritable bowel syndrome. World J Gastroenterol 2013;19:5787-5797.

49. Pourhoseingholi A, Vahedi M, Pourhoseingholi MA, et al. Irritable bowel syndrome, gastro-oesophageal reflux disease and dyspepsia: overlap analysis using loglinear models. Arab J Gastroenterol 2012;13:20-23.

50. Rasmussen S, Jensen TH, Henriksen SL, et al. Overlap of symptoms of gastroesophageal reflux disease, dyspepsia and irritable bowel syndrome in the general population. Scand J Gastroenterol 2015;50:162-169.

51. Smart HL, Nicholson DA, Atkinson M. Gastro-oesophageal reflux in the irritable bowel syndrome. Gut 1986;27:1127-1131.

52. Neumann H, Monkemuller K, Kandulski A, Malfertheiner P. Dyspepsia and IBS symptoms in patients with NERD, ERD and Barrett's esophagus. Dig Dis 2008;26:243-247.

53. Camacho S, Bernal F, Abdo M, Awad RA. Endoscopic and symptoms analysis in Mexican patients with irritable bowel syndrome, dyspepsia, and gastroesophageal reflux disease. An Acad Bras Cienc 2010;82:953-962.

54. Noh YW, Jung HK, Kim SE, Jung SA. Overlap of erosive and non-erosive reflux diseases with functional gastrointestinal disorders according to Rome III criteria. J Neurogastroenterol Motil 2010;16:148-156.

55. Nam SY, Ryu KH, Park BJ. Irritable bowel syndrome is associated with gastroesophageal reflux symptom but not erosive esophagitis. J Neurogastroenterol Motil 2013;19:521-531. 
56. Hsu CS, Liu TT, Wen SH, et al. Clinical, metabolic, and psychological characteristics in patients with gastroesophageal reflux disease overlap with irritable bowel syndrome. Eur $J$ Gastroenterol Hepatol 2015;27:516-522.

57. Mönnikes H, Schwan T, van Rensburg C, et al. Randomised clinical trial: sustained response to PPI treatment of symptoms resembling functional dyspepsia and irritable bowel syndrome in patients suffering from an overlap with erosive gastro-oesophageal reflux disease. Aliment Pharmacol Ther 2012;35:1279-1289.

58. Quigley EM. Non-erosive reflux disease, functional heartburn and gastroesophageal reflux disease; insights into pathophysiology and clinical presentation. Chin J Dig Dis 2006;7:186-190.

59. Malfertheiner $P$, Nocon $M$, Vieth $M$, et al. Evolution of gastro-oesophageal reflux disease over 5 years under routine medical care-the ProGERD study. Aliment Pharmacol Ther 2012;35:154-164.

60. Martinucci I, de Bortoli N, Giacchino M, et al. Esophageal motility abnormalities in gastroesophageal reflux disease. World J Gastrointest Pharmacol Ther 2014;5:86-96.

61. Savarino V, Savarino E, Parodi A, Dulbecco P. Functional heartburn and non-erosive reflux disease. Dig Dis 2007;25:172-174.

62. Zerbib F, Bruley Varannes, Simon M, Galmiche JP. Functional heartburn: definition and management strategies. Curr Gastroenterl Rep 2012;14:181-188.

63. Savarino E, Zentilin P, Mastracci L, et al. Microscopic esophagitis distinguishes patients with non-erosive reflux disease from those with functional heartburn. J Gastroenterol 2013;48:473-482.

64. Hershcovici T, Zimmerman J. Nondigestive symptoms in nonerosive reflux disease: nature, prevalence and relation to acid reflux. Aliment Pharmacol Ther 2008;28:1127-1133.

65. Zimmerman J. Irritable bowel, smoking and oesophageal acid exposure: an insight into the nature of symptoms of gastrooesophageal reflux. Aliment Pharmacol Ther 2004;20:1297-1303.

66. Martinucci I, de Bortoli N, Di Fluri G, et al. Diagnosis of NERD in a population of patients with and without IBS: a pH-MII study. Dig Liv Dis 2011;43:S168. (abstract)

67. Garros A, Mion F, Marjoux S, Damon H, Roman S. Factors associated with nonresponse to proton pump inhibitors therapy in patients referred for esophageal $\mathrm{pH}$-impedance monitoring. Dis Esophagus 2016;29:787-793.

68. Gerson LB, Kahrilas PJ, Fass R. Insights into gastroesophageal reflux disease-associated dyspeptic symptoms. Clin Gastroenterol Hepatol 2011;9:824-833.

69. Min BH, Huh KC, Jung HK, et al. Prevalence of uninvestigated dyspepsia and gastroesophageal reflux disease in Korea: a population-based study using the Rome III criteria. Dig Dis Sci 2014;59:2721-2729.

70. Pleyer C, Bittner H, Locke GR $3^{\text {rd }}$, et al. Overdiagnosis of gastroesophageal reflux disease and underdiagnosis of functional dyspepsia in a USA community. Neurogastroenterol Motil 2014;26:1163-1171.

71. Lee SW, Lee TY, Lien HC, Yeh HZ, Chang CS, Ko CW. The risk factors and quality of life in patients with overlapping functional dyspepsia or peptic ulcer disease with gastroesophageal reflux disease. Gut Liver 2014;8:160-164.

72. Tack J, Caenepeel P, Arts J, Lee KJ, Sifrim D, Janssens J. Prevalence of acid reflux in functional dyspepsia and its association with symptom profile. Gut 2005;54:1370-1376.

73. Gasiorowska A, Poh CH, Fass R. Gastroesophageal reflux disease (GERD) and irritable bowel syndrome (IBS) - is it one disease or an overlap of two disorders? Dig Dis Sci 2009;54:1829-1834.

74. Nastaskin I, Mehdikhani E, Conklin J, Park S, Pimentel M. Studying the overlap between IBS and GERD: a systematic review of the literature. Dig Dis Sci 2006;51:2113-2120.

75. Van Oudenhove L, Demyttenaere K, Tack J, Aziz Q. Central nervous system involvement in functional gastrointestinal disorders. Best Pract Res Clin Gastroenterol 2004;18:663-680.

76. Elsenbruch S. Abdominal pain in Irritable Bowel Syndrome: a review of putative psychological, neural and neuro-immune mechanisms. Brain Behav Immun 2011;25:386-394.

77. Knowles CH, Aziz Q. Visceral hypersensitivity in non-erosive reflux disease. Gut 2008;57:674-683.

78. Costantini M, Sturniolo GC, Zaninotto G, et al. Altered esophageal pain threshold in irritable bowel syndrome. Dig Dis Sci 1993;38:206-212.

79. George AA, Tsuchiyose M, Dooley CP. Sensitivity of the gastric mucosa to acid and duodenal contents in patients with nonulcer dyspepsia. Gastroenterology 1991;101:3-6.

80. Lee KJ, Vos R, Janssens J, Tack J. Influence of duodenal acidification on the sensorimotor function of the proximal stomach in humans. Am J Physiol Gastrointest Liver Physiol 2004;286:G278-G284.

81. Stanghellini V, Barbara G, Cogliandro R, et al. Overlap between GERD and IBS: irrefutable but subtle. J Clin Gastroenterol 2007;41:S114-S117.

82. Jones R, Lydeard S. Irritable bowel syndrome in the general population. BMJ 1992;304:87-90.

83. Gonlachanvit S, Maurer AH, Fisher RS, Parkman HP. Regional gastric emptying abnormalities in functional dyspepsia and gastro-oesophageal reflux disease. Neurogastroenterol Motil 2006;18:894-904.

84. van Lelyveld N, Scheffer R, Mundt M, Samsom M. Partial gastric volumes and upper abdominal sensations in functional dyspeptic and GERD patients: a 3D ultrasonographic study. Am J Gastroenterol 2006;101:1845-1852.

85. de Bortoli N, Natali V, Melissari S, et al. Overlap of GERD and gastrointestinal functional disorders. Minerva Gastroentrol Dietol 2017;63:205-220.

86. Chey WD, Kurlander J, Eswaran S. Irritable bowel syndrome: a clinical review. JAMA 2015;313:949-958.

87. Rubenstein JH, Nojkov B, Korsnes S, et al. Oesophageal hypersensitivity is associated with features of psychiatric disorders and the irritable bowel syndrome. Aliment Pharmacol Ther 2007;26:443-452.

88. Savarino E, Bredenoord AJ, Fox M, Pandolfinoi JE, Roman S, Gyawali CP. International Working Group for Disorders of Gastrointestinal Motility and Function. Expert consensus document: Advances in physiological assessment and diagnosis of GERD. Nat Rev Gastroenterol Hepatol 2017;14:665-676.

89. Numans ME, Lau J, de Wit NJ, Bonis PA. Short-term treatment with proton-pump inhibitors as a test for gastroesophageal reflux disease: a meta-analysis of diagnostic test characteristics. Ann Intern Med 2004;140:518-527.

90. Kaltenbach T, Crockett S, Gerson LB. Are lifestyle measures effective in patients with gastroesophageal reflux disease? An evidence-based approach. Arch Intern Med 2006;166:965-971.

91. Savarino E, de Bortoli N, Zentilin P, et al. Alginate controls heartburn in patients with erosive and nonerosive reflux disease. World J Gastroenterol 2012;18:4371-4378.

92. Zentilin P, Dulbecco P, Savarino E, et al. An evaluation of the antireflux properties of sodium alginate by means of combined multichannel intraluminal impedance and $\mathrm{pH}$-metry. Aliment Pharmacol Ther 2005;21:29-34.

93. Savarino E, Tutuian R, Zentilin $\mathrm{P}$, et al. Characteristics of reflux episodes and symptom association in patients with erosive esophagitis and nonerosive reflux disease: study using combined impedance-pH off therapy. Am J Gastroenterol 2010;105:1053-1061.

94. Cremonini F, Ziogas DC, Chang HY, et al. Meta-analysis: the effects of placebo treatment on gastro-oesophageal reflux disease. Aliment Pharmacol Ther 2010;32:29-42. 
95. Labenz J, Malfertheiner P. Treatment of uncomplicated reflux disease. World J Gastroenterol 2005;11:4291-4299.

96. Chiba N, De G CJ, Wilkinson JM, et al. Speed of healing and symptom relief in grade II to IV gastroesophageal reflux disease: a meta-analysis. Gastroenterology 1997;112:1798-1810.

97. Sigterman KE, van Pinxteren B, Bonis PA, Lau J, Numans ME. Short-term treatment with proton pump inhibitors, $\mathrm{H} 2$-receptor antagonists and prokinetics for gastro-oesophageal reflux disease-like symptoms and endoscopy negative reflux disease. Cochrane Database Syst Rev 2013;5:CD002095.

98. Savarino E, Marabotto E, Zentilin P, et al. The added value of impedance-pH monitoring to Rome III criteria in distinguishing functional heartburn from non-erosive reflux disease. Dig Liver Dis 2011;43:542-547.

99. Robinson M, Sahba B, Avner D, Jhala N, Greski-Rose PA, Jennings DE. A comparison of lansoprazole and ranitidine in the treatment of erosive oesophagitis. Multicentre Investigational Group. Aliment Pharmacol Ther 1995;9:25-31.

100. Galmiche JP, Barthelemy P, Hamelin B. Treating the symptoms of gastro-oesophageal reflux disease: a double-blind comparison of omeprazole and cisapride. Aliment Pharmacol Ther 1997;11:765-773.

101. Vantrappen G, Rutgeerts L, Schurmans P, Coenegrachts JL. Omeprazole $(40 \mathrm{mg}$ ) is superior to ranitidine in shortterm treatment of ulcerative reflux esophagitis. Dig Dis Sci 1988;33:523-529.

102. Zerbib F, Belhocine $\mathrm{K}$, Simon $\mathrm{M}$, et al. Clinical, but not oesophageal $\mathrm{pH}$-impedance, profiles predict response to proton pump inhibitors in gastro-oesophageal reflux disease. Gut 2012;61:501-506.

103. Mönnikes H, Heading RC, Schmitt H, Doerfler H. Influence of irritable bowel syndrome on treatment outcome in gastroesophageal reflux disease. World $J$ Gastroenterol 2011;17:3235-3241.

104. Weijenborg PW, Cremonini F, Smout AJ, Bredenoord AJ. PPI therapy is equally effective in well-defined non-erosive reflux disease and in reflux esophagitis: a meta-analysis. Neurogastroenterol Motil 2012;24:747-757, e350.

105. Schindlbeck NE, Klauser AG, Berghammer G, Londong W, Müller-Lissner SA. Three year follow up of patients with gastrooesophageal reflux disease. Gut 1992;33:1016-1019.

106. Carlsson R, Dent J, Watts R, et al. Gastro-oesophageal reflux disease in primary care: an international study of different treatment strategies with omeprazole. International GORD Study Group. Eur J Gastroenterol Hepatol 1998;10:119-124.

107. Talley NJ, Lauritsen K, Tunturi-Hihnala H, et al. Esomeprazole $20 \mathrm{mg}$ maintains symptom control in endoscopy-negative gastro-oesophageal reflux disease: a controlled trial of 'ondemand' therapy for 6 months. Aliment Pharmacol Ther 2001;15:347-354.

108. Pace F, Tonini M, Pallotta S, Molteni P, Porro GB. Systematic review: maintenance treatment of gastro-oesophageal reflux disease with proton pump inhibitors taken 'on-demand'. Aliment Pharmacol Ther 2007;26:195-204.

109. Wu JC, Lai LH, Chow DK, Wong GL, Sung JJ, Chan FK. Concomitant irritable bowel syndrome is associated with failure of step-down on-demand proton pump inhibitor treatment in patients with gastro-esophageal reflux disease. Neurogastroenterol Motil 2011;23:155-160, e31.

110. de Bortoli N, Natali V, Melissari S, et al. Association between baseline impedance values and response proton pump inhibitors in patients with heartburn. Clin Gastroenterol Hepatol 2015;13:1082-1088.e1.
111. Grover M, Drossman DA. Psychopharmacologic and behavioral treatments for functional gastrointestinal disorders. Gastrointest Endosc Clin N Am 2009;19:151-170.

112. Surdea Blaga T, Dumitrascu D, Galmiche JP, Bruley des Varannes S. Functional heartburn: clinical characteristics and outcome. Eur J Gastroenterol Hepatol 2013;25:282-290.

113. Viazis N, Karamanolis G, Vienna E, Karamanolis DG. Selectiveserotonin reuptake inhibitors for the treatment of hypersensitive esophagus. Therap Adv Gastroenterol 2011;4:295-300.

114. Talley NJ. A unifying hypothesis for the functional gastrointestinal disorders: really multiple diseases or one irritable gut? Rev Gastroenterol Disord 2006;6:72-78.

115. Talley NJ. Overlapping abdominal symptoms: why do GERD and IBS often coexist? Drugs Today (Barc) 2006;42 Suppl B:3-8.

116. Guillemot F, Ducrotté P, Bueno L. Prevalence of functional gastrointestinal disorders in a population of subjects consulting for gastroesophageal reflux disease in general practice. Gastroenterol Clin Biol 2005;29:243-246.

117. Raftopoulos Y, Papasavas P, Landreneau R, et al. Clinical outcome of laparoscopic antireflux surgery for patients with irritable bowel syndrome. Surg Endosc 2004;18:655-659.

118. Kountouras J, Chatzopoulos D, Zavos C, Boura P, Venizelos J, Kalis A. Efficacy of trimebutine therapy in patients with gastroesophageal reflux disease and irritable bowel syndrome. Hepatogastroenterology 2002;49:193-197.

119. Kinoshita Y, Miwa H, Sanada K, Miyata K, Haruma K. Clinical characteristics and effectiveness of lansoprazole in Japanese patients with gastroesophageal reflux disease and dyspepsia. J Gastroenterol 2014;49:628-637.

120. Miwa H, Haruma K, Sakamoto S, Sanada K, Hiroi S, Kinoshita Y. Demography and treatment response in patients with predominant non-erosive reflux disease or functional dyspepsia. J Gastroenterol Hepatol 2015;30:834-841.

121. Bürgel N, Bojarski C, Mankertz J, Zeitz M, Fromm M, Schulzke JD. Mechanisms of diarrhea in collagenous colitis. Gastroenterology 2002;123:433-443.

122. Keszthelyi D, Dackus GH, Masclee GM, Kruimel JW, Masclee AA. Increased proton pump inhibitor and NSAID exposure in irritable bowel syndrome: results from a case-control study. BMC Gastroenterol 2012;12:121.

123. Mullin JM, Valenzano MC, Whitby M, et al. Esomeprazole induces upper gastrointestinal tract transmucosal permeability increase. Aliment Pharmacol Ther 2008;28:1317-1325.

124. Lombardo L, Foti M, Ruggia O, Chiecchio A. Increased incidence of small intestinal bacterial overgrowth during proton pump inhibitor therapy. Clin Gastroenterol Hepatol 2010;8:504-508.

125. Ulluwishewa D, Anderson RC, McNabb WC, Moughan PJ, Wells JM, Roy NC. Regulation of tight junction permeability by intestinal bacteria and dietary components. $J$ Nutr 2011;141:769-776.

126. Keszthelyi D, Jansen SV, Schouten GA, et al. Proton pump inhibitor use is associated with an increased risk for microscopic colitis: a case-control study. Aliment Pharmacol Ther 2010;32:1124-1128.

127. Bassett JT, Cash BD. A review of irritable bowel syndrome and an update on therapeutic approaches. Expert Opin Pharmacother 2008;9:1129-1143.

128. Creed F, Fernandes L, Guthrie E, et al. The cost-effectiveness of psychotherapy and paroxetine for severe irritable bowel syndrome. Gastroenterology 2003;124:303-317.

129. Tack J, Broekaert D, Fischler B, Van Oudenhove L, Gevers AM, Janssens J. A controlled crossover study of the selective serotonin reuptake inhibitor citalopram in irritable bowel syndrome. Gut 2006;55:1095-1103. 